\title{
Editorial
}

\section{The law of requisite variety: Is it applicable to human systems?}

It is rarely disputed that we live in a world of increasing complexity. Some would even say: 'Entropy is rising; negentropy is falling', or 'Proliferating variety is a measure of increasing chaos', and such.

This is too simple. The increase in complexity should refer to the increase in organized complexity, not to chaos. Even chaos itself is now seen as a kind of organization which is still incomprehensible or inaccessible to humans.

Some insist that 'only variety can absorb variety' and call it the great 'law' of nature. Matching, or requisite, variety is then proposed as the condition of successful regulation and control in our increasingly complex social systems.

This is all referred to as Ashby's Law of Requisite Variety and because it is a law it seems to have been obeyed by generations of cyberneticians, systems engineers and management theorists.

Variety is defined as the number of possible states of a system. If it is either off or on - its variety is 2 . When you wish to control a system, you have to control its variety, i.e., the states it can assume. The need for such control is self-evident: some of the states are 'desirable', others are not. That could serve as a definition of control: ability to elicit 'desirable' states.

It is important to remember that Ross Ashby derived the law of requisite variety from contemplating matrices of the two-person, scalar-outcome, competitive parlor games of von NeumannMorgenstern. In this sense his law must be correct and has no empirical or scientific hypothesis dimensions. It's like saying that one cannot form a

North-Holland

Human Systems Management 6 (1986) 269-271 square by arranging three objects. Or, in Ashby's own assessment:

'The theorem is primarily a statement about possible arrangements in a rectangular table. It says that certain types of arrangement cannot be made' [1, p. 209].

This is fair and square. Elsewhere Ashby refers to this law as 'trite in the simple cases', 'intuitively obvious and hardly deserving statement', and 'the law owes nothing to experiment'.

Another version of the law says that system's capacity as a regulator cannot exceed is capacity as a channel of communication (communication in the sense of Shannon).

Given the self-evident nature of Ashby's Law, the next question is: are complex social systems meaningfully expressible in terms of two-dimensional tables? That is, can a trivial algebraic property be extended into successful regulation of the most complex social domain of human interactions?

Norbert Wiener expressed his profound skepticism:

'...they [Wiener's anthropologist friends] consider that the main task of the immediate future is to extend to the fields of anthropology, of sociology, of economics, the methods of the natural sciences, in the hope of achieving a like measure of success in the social fields. From believing this necessary, they come to believe it possible. In this, I maintain, they show an excessive optimism, and a misunderstanding of the nature of all scientific achievement' (emphasis added) [3, p. 162].

Even Ashby expressed his doubts and ambiguity on the issue:

'These regulations [of large and complex systems] ... at first sight they look so different that one may well wonder whether what has been said so far is not essentially inapplicable' [1, p. 244]. 
'It is commonly said that Hitler's control over Germany was total. So far as his power of regulation was concerned, the law says that his control amounted to just 1 man-power, and no more. (Whether this statement is true must be tested by the future; its chief virtue now is that it is exact and uncompromising.)' [1, p. 213.]

If the law is self-evident and 'owes nothing to experiment' how can it be 'tested by the future'? What does it mean and how useful is it to say that 'Hitler's control amounts to 1 man-power'? And why is not the requisite 'willingness of the masses to be controlled' even postulated in sociocybernetics?

Neither Wiener nor Ashby were experienced or even interested in dealing with social systems. It is only their later interpreters who made the arching leaps which the founders never cared to make.

Stafford Beer presents the following reasoning:

'Consider a labour force of ten men, operating under the rule that anyone who did not immediately do what he was told would be shot. This system has a variety of ten, and is readily controlled by anyone with a gun and ten bullets. Now relax the constraint that disobedience means death. At once the variety of this ten-man team rises to something approaching infinity. These men may now do anything at all: obey orders, disobey orders, vary orders, ignore orders. Or they go away and play cards' [2, p. 110].

Experience tells us that the above system can be equally readily controlled not only by a gun and ten bullets, but also by a gun and one bullet, by a gun and no bullets and even by somebody with no gun and no bullets. When you relax the constraint, team variety does not rise to infinity and men do not (and even cannot) do whatever they wish: most likely they organize themselves for a very specific and highly predictable action. Their variety may even decrease after the gun is removed: the man with a gun can command them to eat, dance, write poetry, sing, work, lie down, drink or do consulting. With no such constraint, they might be capable of playing cards only. To say that a team of ten men has a 'variety of ten', whether true or not, is quite irrelevant and empty for the purposes of management.

Humans do not exist outside organized interdependence and their response variety is, if anything, too limited and constrained. Our problem is not with further limiting this variety, but rather with enhancing it to more desirable levels.

Even in non-human biological systems, to the extent that they are social systems, Ashby's law applicability is not clear.

Ashby is confident here:

'A species continues to exist primarily because its members can block the flow of variety (thought of as disturbance) to the gene-pattern, and this blockage is the species' most fundamental need' [1, p. 212].

Such statement is self-evident: obviously any disturbance threatening the survival of the genepattern must be blocked - by definition. To make it less trivial, Ashby then introduces the notion of transforming (or re-coding) some disturbances into information, which is of course useful and should be made as large as possible.

Without pursuing this further, the fact is that biological communities thrive on and are effectively 'controlled' by the continuous inflow of perturbations. As long as some form of perturbation occurs (and some may be quite damaging), there is an opportunity for all members of a competitive hierarchy to thrive in a locality, thus preserving a degree of biodiversity. Monocultures are rare in nature.

Regulation is possible by: (a) re-defining acceptability and lowering of standards (trivial); (b) increasing the scope and power of the regulator until adequate capacity is achieved (most difficult or impossible); (c) discovering 'inner' constraints on the variety of disturbance and turning them to good use (important and useful, regulator's only hope).

The judgments in parentheses are Ashby's own.

Because social systems are organized systems and their 'components' are heavily interdependent, their variety can be reduced by discovering or imposing constraints; also, for the same reasons, by increasing their interdependence.

Example. Group of workers performs a task and is controlled by a manager. In order to maximize their individual performance, they are specialized into small subtasks and they are 'buffered' against each other's errors or slippages by stocks of inventoried parts. They are an aggregate rather than a team (or system), they are relatively independent of each other, and they are increasingly difficult to control. In fact, increasing the size of such aggregate (through progressive division of labor) requires even faster growth in the coordinative 
hierarchy of control - i.e., management. Matching variety by variety leads to overblown bureaucracy and thus to virtually uncontrollable complexity.

Assume that instead of the above approach we take the opposite road: we remove buffers and inventories and thus increase workers' interdependence; we allow them to join in circles and find their own ways of solving problems; we enhance their flexibility and ability to cooperate by allowing them to overstep their narrow specializations; we reward them as a team, not only for task performance but also for effort coordination (self-management and self-control).

As the self-controlling team is emerging we can solidify its self-regulatory abilities by introducing appropriately amplifying technology which would allow them to function without any central controller or agent. Then we can start dismantling the 'variety-matching' hierarchy and bureaucracy of control.

In other words, in human and social systems there exists organized interdependence which, if amplified and consciously enhanced, can lead to more effective self-regulation.

Progressive and successful businesses know this: quality circles, communication interflow, just-intime, multifunctionality and job rotation, co-determination, co-ownership, participative management, autonomy, self-management, and generalpurpose flexible technology are their 'tools of trade'.

Traditional and declining businesses do not know it: multilayered hierarchies of command, large staffs, bureaucracy, overspecialization, centralized data and information systems, complex control and accounting systems, rigidity and singleness of purpose, dedicated technology and Challenger-type organization and culture are their 'tools of trade'.

It appears that this sort of 'variety engineering' (with, for example, algedonic-feedback cyberstrides, using Bayesian statistical theory) increases rather than decreases system's regulatory complexity.

Our human productive systems do not need to be further regulated, controlled and 'engineered'. Just the opposite: they have to become freer, more autonomous, more flexible, and more innovative we all need more of their 'product'. It is the military and governmental systems which are in need of stricter controls: because, after all, control and regulation is their 'product'. More 'productivity' here leads to ever stricter and ever more effective regulative controls of the society.

In dealing with social complexity, we can enhance and amplify human autonomy, self-management, creativity, innovation, self-reliance and self-organization; or we can try computer-based and feedback-ridden regulatory mechanisms of matching complexity, train more skillful regulators and controllers (and more of them), and improve the control efficiency of organizational hierarchies.

In the first case, we have to understand the systems: what they want and what they are striving for; we (as regulators) are the means. In the second case, we have to understand ourselves (as regulators): what we want and what we are striving for; human systems are the means.

Both approaches are possible, but not equally ethical or desirable.

\author{
Milan Zeleny \\ Fordham University \\ at Lincoln Center \\ New York, NY 10023, USA
}

\section{References}

[1] Ashby, W. Ross, An introduction to cybernetics (Wiley, Science Editions, New York, 1963).

[2] Beer, Stafford, Platform for change (Wiley, New York, 1975).

[3] Wiener, Norbert, Cybernetics: Or control and communication in the animal and the machine (MIT Press, Cambridge, MA, 1961). 